\title{
Imaging fibre bundles for Fizeau-based optical coherence tomography
}

\author{
G. F. Sarantavgas, H. D. Ford and R. P. Tatam \\ Engineering Photonics Group, School of Engineering, Cranfield University, Cranfield, Bedford, \\ MK43 0AL, UK.
}

\begin{abstract}
An OCT system incorporating a coherent fibre imaging bundle is described. Fibres are accessed sequentially by a beam focused onto the input face of the bundle, allowing 2D or 3D images to be acquired using point detection. A Fizeau interferometer configuration is used, in which light from the distal end of a fibre in the bundle (forming the reference arm) mixes with light reflected by the sample itself (forming the sample arm). The use of coherent imaging bundles for OCT beam delivery allows mechanical scanning parts to be removed from the sample arm, resulting in a passive probe. Such a configuration can form a compact, robust and "downlead insensitive" OCT system. In the common-path configuration used, an inherent path-length difference is present in the Fizeau sample interferometer, so an additional processing interferometer is required to ensure path-length matching. The depth scanning mechanism is confined within the processing interferometer, external to the sample probe.
\end{abstract}

Keywords: Optical Coherence Tomography, Fibre bundle, Fibre bundle OCT, Fizeau interferometer based OCT, Biomedical imaging, OCT probe, "down-lead" insensitive OCT, Low coherence interferometry, Fizeau interferometer based fibre bundle OCT, Common path OCT.

\section{INTRODUCTION}

Typically, an optical coherence tomography (OCT) system is configured using an in-fibre Michelson interferometer ${ }^{1}$, with a reference reflector in one interferometer arm, and the sample in the other. However, the stability of this commonly-used arrangement is environmentally sensitive. Variations in the deployment of the interferometer fibres cause polarization-state changes which are different for the two arms. Signal fading therefore occurs, reducing the amplitude of the fringes ${ }^{2}$. This is not a severe problem if the sensitivity drift is slow, since the visibility value will exceed 0.5 during about $70 \%$ of the measurement time. However, polarization changes within the duration of an A-scan, such as might be experienced during endoscopic manipulation, are more of a problem. Variations on this timescale can introduce artefacts into the OCT image, in the form of spurious interfaces or regions of missing data ${ }^{3}$. The use of a Fizeau interferometer, as shown for a time-domain configuration in figure 1, eliminates polarisation fading problems, since the reference and signal beams travel in the same fibre. A Fizeau-type OCT system using lock-in detection was originally reported by Bamford ${ }^{4}$, and alternative configurations subsequently investigated ${ }^{5,6}$. Several other groups have recently reported investigations of common-path configurations ${ }^{7,8}$.

In a Fizeau-type OCT system, the reference reflection is derived either from a glass plate positioned close to the sample or, preferably, from the reflection at the end of the delivery fibre. This removes the requirement to suppress the fibre end reflection. The selected reference reflectivity affects system sensitivity. The usual $4 \%$ fibre end reflectivity is quite appropriate for typical reflectivities expected from biological samples but, if required, the fibre end can be coated with titanium dioxide to increase the reflectivity up to a value of around $30 \%{ }^{9}$. The reference and signal beams traverse a common path within the optical fibre section of the interferometer, experiencing identical phase/polarisation perturbations. The state of polarisation for both beams is matched at all positions, and the visibility of any interference seen at the detector is maintained at the maximum for the system.

Coherence Domain Optical Methods and Optical Coherence Tomography in Biomedicine XII, edited by Joseph A. Izatt, James G. Fujimoto, Valery V. Tuchin, Proc. of SPIE Vol. 6847, 68470C, (2008) $1605-7422 / 08 / \$ 18 \cdot$ doi: $10.1117 / 12.762640$ 
Because of the path length imbalance inherent to Fizeau systems, a processing interferometer is required, to readjust the overall path length difference to a value within the source coherence length. A Michelson-type processing interferometer is shown in figure 1.

The sensitivity of the system is defined by the signal-to-noise ratio (SNR), which affects the depthdependent contrast seen in the OCT images, and therefore both the maximum depth at which acceptable imaging can be achieved and the minimum change in refractive index that can be detected. SNR is typically lower in Fizeau-type OCT systems than in those based on Michelson interferometers, because there is a larger dc background, resulting from light that does not participate in the interference process ${ }^{10}$. The excess noise term arising from this cannot be cancelled using balanced detection, because the noise on the two complementary outputs from the Mach-Zehnder interferometer is out of phase, as is the ac signal term ${ }^{7}$.

However, there are advantages to the Fizeau approach. The fibre end reflection, which in a Michelson-type OCT system is a source of noise, is now used instead as the system reference beam. The 'downlead insensitivity', conferred by the common-path arrangement in the probe arm, maintains the SNR at its maximum possible value and in some situations prevents the occurrence of polarisation-fading artefacts in OCT images. In the more typicallyused Michelson OCT configuration, recoupling into single-mode fibre must be achieved from a scanned reference beam, which can be awkward. The Fizeau configuration avoids reference beam recoupling.

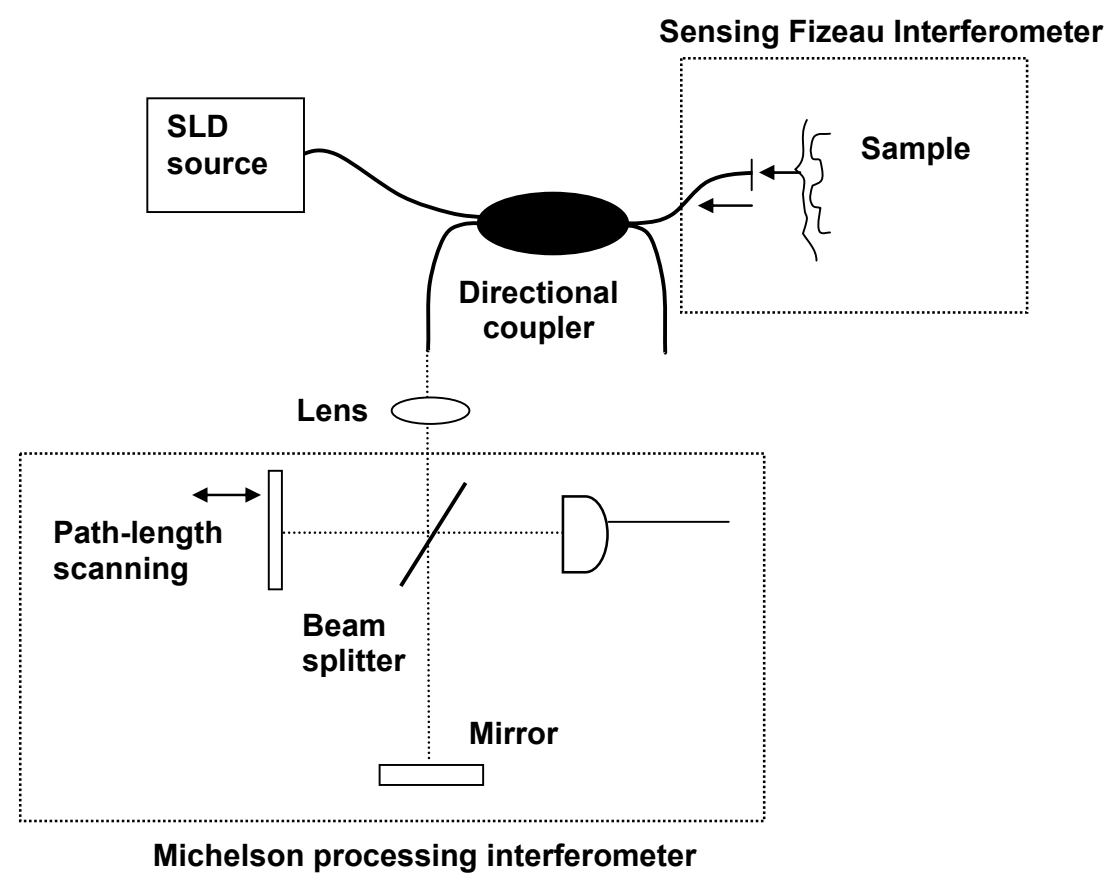

Fig. 1. Configuration for Fizeau configuration OCT system with a Michelson processing interferometer.

In OCT, 3D imaging is generally achieved by scanning the probe beam across the surface of the sample in a raster pattern, acquiring an A-scan at each position and providing images of $1-30 \mu \mathrm{m}$ resolution ${ }^{11}$ at imaging depths of $1-2 \mathrm{~mm}^{12}$. Currently, to implement this technique, active scanning components are required at the probe tip. This is relatively straightforward in a bulk-optic system, but more difficult in a fibre-optic endoscope probe, where miniaturized scanning components are necessary ${ }^{13,14,15}$. In this paper we discuss how the incorporation of a coherent imaging bundle, in a Fizeau based interferometer configuration, can allow a 'downlead insensitive' OCT probe to be designed, with the elimination of scanning mechanical parts from the probe.

We have previously demonstrated an OCT probe incorporating an imaging fibre bundle, in which all fibres of the bundle were illuminated simultaneously, with a CCD camera used for image capture ${ }^{16}$. The present paper 
describes an alternative methodology, in which each fibre is illuminated in turn, using scanning components at the input to the bundle to address the individual fibres. This system enables point detectors to be used instead of a camera, allowing high-bandwidth noise filtering techniques to be applied to the detector output.

Imaging bundles have been used successfully for OCT in a Michelson configuration. However, the state of polarization of light varies considerably for light in different fibres of the bundle which, at any one time, will result in non-optimal interference signals for many of the fibres, as shown in figure 2.

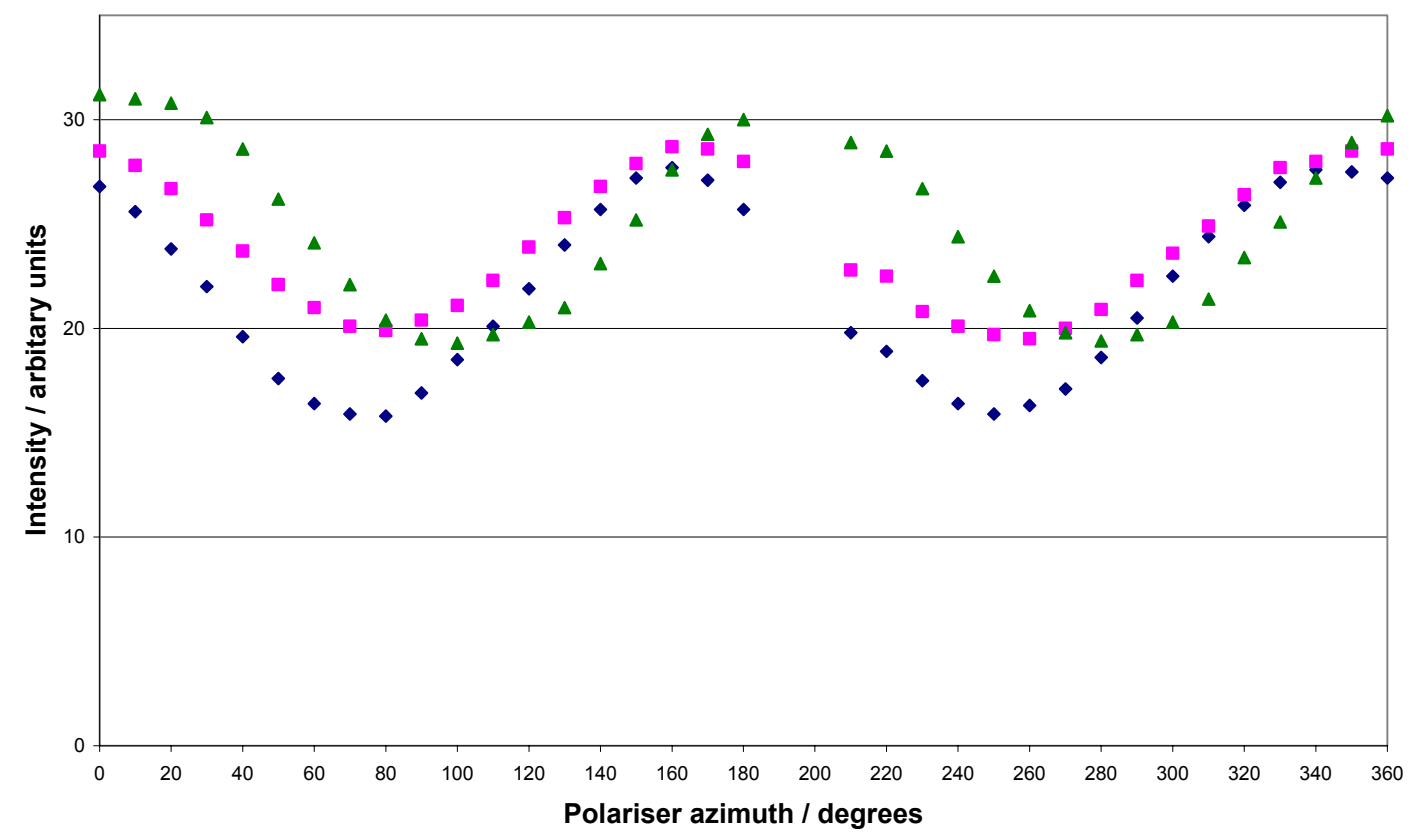

Fig. 2. Plot of intensity variation in the output from three individual fibres of the bundle, as a polarizer is rotated at the output, showing that the state of polarization differs between fibres.

It is not easy to see how the polarization of light in each individual fibre can be manipulated. However, in the Fizeau configuration, the bundle does not form part of either interferometer; therefore the instrument is "downlead insensitive" ${ }^{18}$.

\section{EXPERIMENTAL SYSTEM BASED ON A FIZEAU CONFIGURATION}

In our experimental arrangement, shown in figure 3, the coupler was replaced by a polarisation-independent optical circulator, which greatly increases the optical efficiency. The source was a pigtailed super-luminescent diode (Covega) centred at $1550 \mathrm{~nm}$, with a full-width half maximum (FWHM) of $55 \mathrm{~nm}$, and an output power of $9.5 \mathrm{~mW}$ following the fibre pigtail. Light from the source is routed through the circulator from one port to the next, with a consequent transfer loss of only a few percent. Thus, neglecting losses, all light from the broadband source is delivered by the optical circulator to the Fizeau sensing interferometer. An optical power of $7.6 \mathrm{~mW}$ was measured onto the sample. Both the fibre end-reflection and the back-scattered light from the sample are then directed to the bulk-optic Mach-Zehnder balancing interferometer, where the light is collimated using a fibre-connected GRIN lens and then amplitude-divided by a plate beam splitter. Plate beam splitters are used in the bulk-optic part of the system because their polarisation sensitivity is lower than that of cube beam splitters. One surface of each beam splitter is anti-reflection coated to prevent "ghosting". In one arm of the interferometer, a glass $45^{\circ}$ prism is mounted on a computer-controlled translation stage, which allows both adjustment of the gross path length difference, to match 
that in the Fizeau interferometer, and high-resolution A-scanning. The beam offset introduced by the prism allows all the retro-reflected light to continue through the interferometer, and thus minimises losses. A second $45^{\circ}$ prism acts as a reflector in the other interferometer arm. In principle, only one of the interferometer arms need include a prism, but the symmetrical arrangement using two prisms is better for minimisation of dispersion effects. There is an overall loss of $50 \%$ in the processing interferometer.

The two interferometer beams recombine at the second beam splitter. The signal is acquired by an InGaAs photodetector and digitised using a 200,000 samples s-1, 16-bit A/D card housed within a PC. An A-scan obtained using a mirror in the sample arm produces a coherence function with a FWHM value of $19 \mu \mathrm{m}$ in air, or about $13 \mu \mathrm{m}$ in a typical biological sample. This defines the longitudinal resolution of the system. The PI translation stage used has a maximum scanning speed of $1.7 \mathrm{~mm} \mathrm{~s}-1$, and is controlled by means of a LabviewTM program.

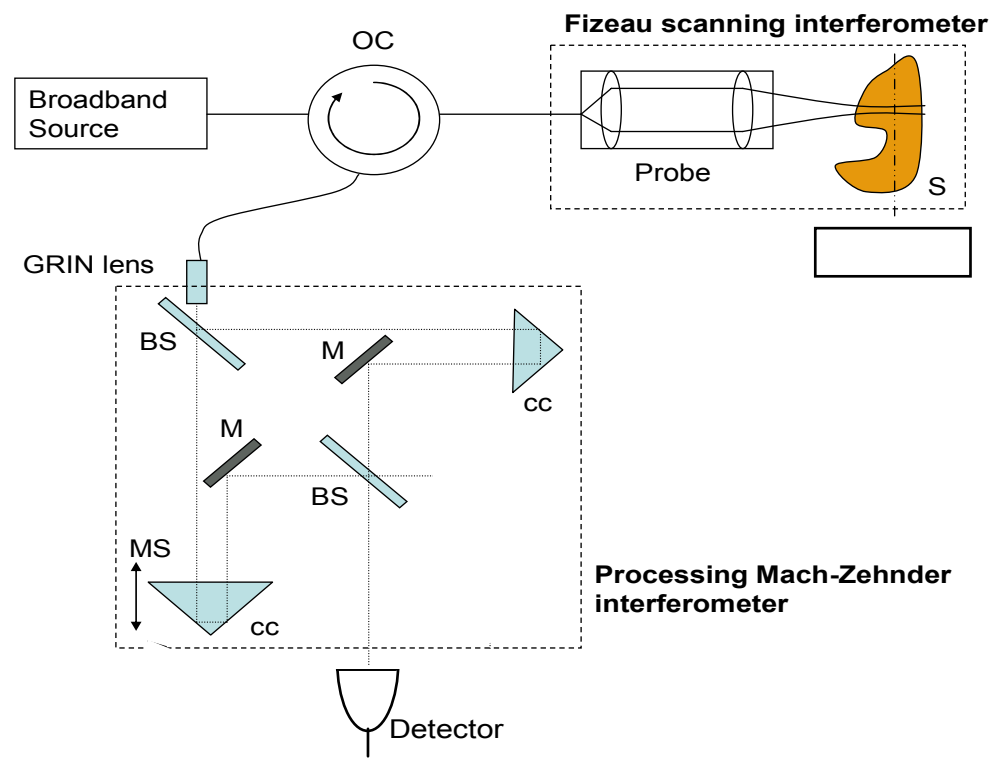

Fig. 3. Configuration for Fizeau-based OCT system with Mach-Zehnder type processing interferometer. OC $=$ optical circulator, $\mathrm{S}=$ sample, $\mathrm{BS}=$ beam splitter, $\mathrm{M}=$ mirror, $\mathrm{CC}=$ corner cube prism, $\mathrm{MS}=$ micropositioning stage.

The probe-end focusing head comprises a pair of lenses of focal lengths 20 and $40 \mathrm{~mm}$ respectively, giving a theoretical beam waist of about $23 \mu \mathrm{m}$ in air, and a Rayleigh range of around $540 \mu \mathrm{m}$. Scanning can typically be performed through about three Rayleigh ranges, centred on the position of best focus, without severe loss of SNR or spatial resolution. Therefore, for this system, the accessible depth as defined by the Rayleigh range is about 1.5-2 $\mathrm{mm}$. In a high-index medium, the beam waist and the Rayleigh range are increased; for a material of index 1.5, the observed performance of this system is expected to be reasonably constant within a sample depth range of about 3 $\mathrm{mm}$.

\subsection{Tests using oils of calibrated refractive index}

The sensitivity of the system was assessed using Cargille oils of extremely well calibrated refractive index, deposited on a glass microscope slide of $1 \mathrm{~mm}$ thickness. Measurements were performed with the OCT probe mounted vertically, as shown in figure 4. A cylindrical collar, adjustable in height, was fixed around the probe end such that the upper end of the cylinder could be positioned at the best focus distance from the lenses and used as a platform for mounting the oil samples (figure 4). This provided a repeatable measurement system, where the 
oil/glass interface was at precisely the same distance from the probe end on each occasion, to within a few microns. Addressing the sample with the beam coming from below the slide ensured that the beam focus position was not altered by travelling through samples of differing thickness and/or refractive index.

The Fresnel equations yield the wavelength-dependent transmission and reflectivity for an interface between two isotropic dielectric media. For normal incidence, the reflectivity equation simplifies to

$$
R=\left(\frac{n_{\text {glass }}-n_{\text {oil }}}{n_{\text {glass }}+n_{\text {oil }}}\right)^{2}
$$

Thus the reflectivity of an interface can be calculated. For the microscope slide, the RI was calculated from the ratio of optical thickness, measured using the OCT system as $1.5211 \mathrm{~mm}$, to the actual physical thickness, measured using vernier calipers as $0.999 \mathrm{~mm}$. This yielded a RI of $1.523( \pm 0.01)$ at $1550 \mathrm{~nm}$. For the Cargille oils used, the RI was quoted at $588.3 \mathrm{~nm}$, and was converted to $1550 \mathrm{~nm}$ in each case using the Cauchy equation provided with the bottles. The RI values obtained at $1550 \mathrm{~nm}$ were generally about 0.01 lower than the quoted values at $588.3 \mathrm{~nm}$, and the accuracy of the calculated value at $1550 \mathrm{~nm}$ was about 0.007 according to manufacturer specifications.

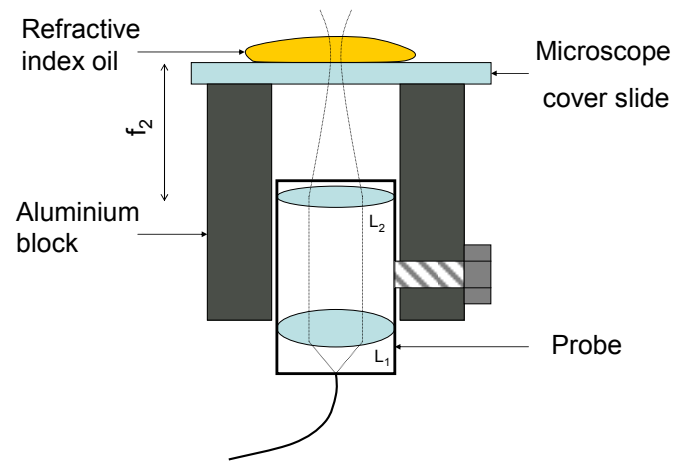

Fig 4. Experimental arrangement for reflectivity measurements on calibrated refractive-index oils. $\mathrm{L}=$ lens. The distance $\mathrm{f} 2$ corresponds to the focal length of the second lens in the probe head.

The lower surface of the cover slip acts as a useful reference surface for the measurements, since the OCT peak obtained for this glass/air interface should be the same on each occasion. The sample position was set by placing a cover slip on the platform, and adjusting the mount position until the OCT signals received for the upper and lower surfaces of the slip were of approximately equal intensity. Measurements were then made for a series of oils with indices ranging from 1.400-1.600. The total reflected power was assumed to result from the sum of reflections at the air/glass and glass/oil interfaces. The oil/air interface is more than one Rayleigh range from best focus and its contribution to the total reflected signal is small.

For each of the oils, the SNR of the glass/oil signal peak was assessed. This was obtained from the ratio of the peak amplitude to the standard deviation of the noise amplitude.

$$
S N R_{\text {glass / oil }}=20 \log _{10}\left(\frac{A_{\text {glass /oil }}}{\sigma_{\text {noise }}}\right)
$$


The results are plotted in figure 5. As expected, the SNR decreases as the index of the oil asymptotically approaches that of the substrate. The smallest index difference measured in this experiment was about 0.01 , which resulted in an SNR of $35.5 \mathrm{~dB}$ measured directly from the detector output, or $64.5 \mathrm{~dB}$ when software filtering was applied.

Differences in RI between regions within a biological sample tend to be in the second or third decimal place. The results from the oil measurements suggest that the SNR obtained from our system is sufficient for goodquality imaging from samples with this level of RI variation.

This system used time-domain processing, but it would also be possible to operate the system as a Fourierdomain instrument ${ }^{17}$ by fixing the path-length in the processing interferometer and dispersing the output signal onto a spectrometer. This inevitably leads to a relatively large dc background, and it would be preferable to dispense with the processing interferometer altogether. However, the path-length inherent to the Fizeau interferometer is too large to allow for Fourier-domain operation in this mode, unless an external reflector is used to generate the reference reflection ${ }^{8}$.

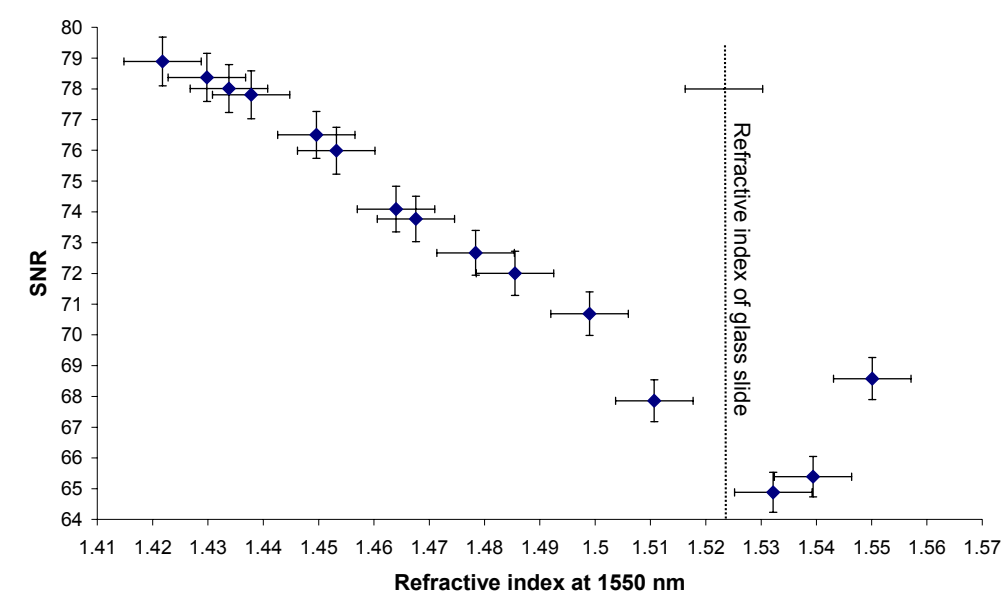

Fig. 5. SNR of the OCT signal for a series of oils of known refractive index, deposited on a glass microscope slide.

\section{FIZEAU INTERFEROMETER CONFIGURATION USING COHERENT IMAGING FIBRE BUNDLES}

The experimental arrangement for the OCT system is described below. It is a bundle-based analogue of the single-point version. As shown in figure 6, light emanating from an SLD at $830 \mathrm{~nm}$, with $9.86 \mu \mathrm{m}$ coherence length is collimated, using an aspheric lens to ensure diffraction limited performance. 


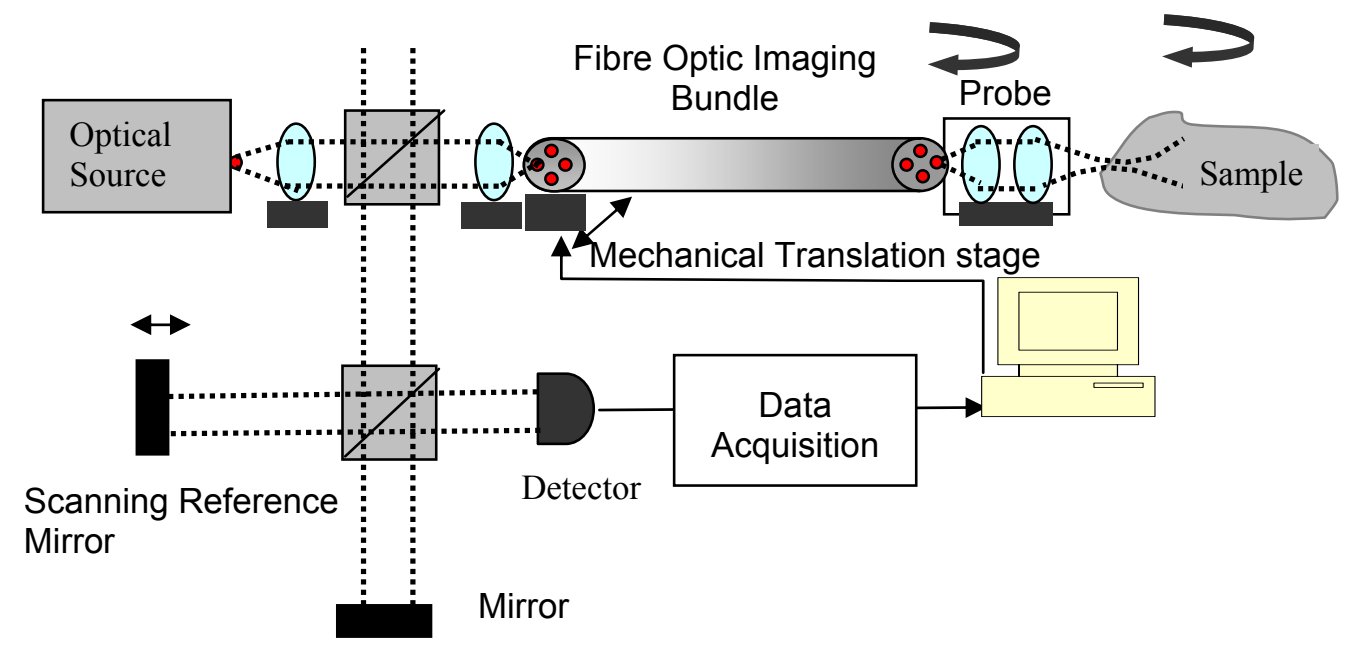

Fig. 6. Fizeau based OCT incorporating a coherent imaging fibre optic bundle.

The collimated beam is focused onto the input face of the bundle, by means of a second aspheric lens providing a focused beam diameter of $4.4 \mu \mathrm{m}$. The input end of the bundle was polished at an angle of 8 degrees to avoid any back reflection to the processing interferometer. The length of the bundle was $1.1 \mathrm{~m}$ and was comprised 15 000 fibres. Each fibre in turn was addressed by the focused input beam. The beam is transmitted through the bundle and is focused onto the sample by the probe lenses, also comprising appropriate aspherics. The sample beam is focused with a beam diameter of about $8.2 \mu \mathrm{m}$ and a Rayleigh range of about $250 \mu \mathrm{m}$. The Fizeau interferometer is formed between reflected light from the distal end of a fibre in the bundle (forming the reference arm) and light reflected by the sample itself (forming the sample arm). Since an inherent path length mismatch is present, a processing interferometer is required either in the form of a Michelson or Mach-Zehnder. A bulk-optic processing interferometer is preferred to avoid reintroducing polarization problems and to minimize dispersion. To address each fibre in the bundle in succession, the bundle was mounted onto a computer controlled mechanical translation stage, which was scanned across the focused input beam. Two coherent fibre optic imaging bundles used were tested which were provided by Schott North America. The packing arrangement of fibres in the bundle, as shown in figure 7, can be either a regular hexagonal pattern for leached bundles, or an arrangement of square packed $5 \times 5$ sub-bundles for wound bundles, with fibre centre spacings of $10 \mu \mathrm{m}$ and core diameters of $8 \mu \mathrm{m}$.

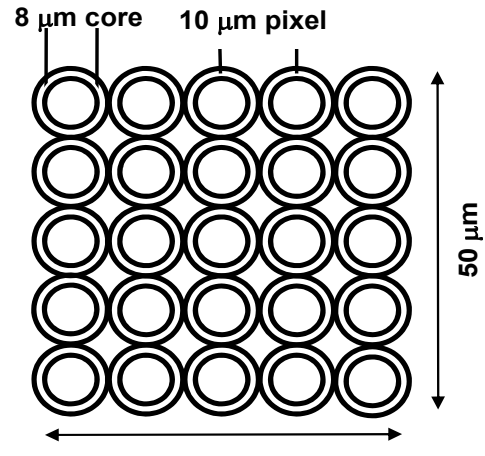

$50 \mu \mathrm{m}$

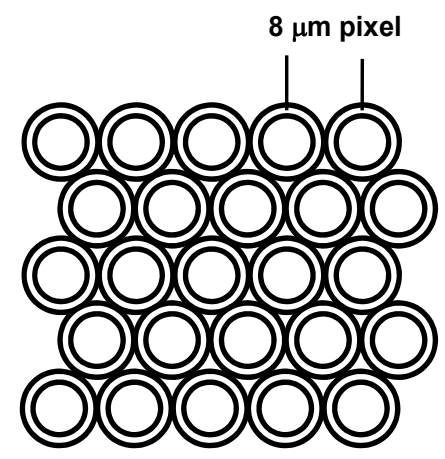

Fig. 7. Fibre bundle packaging arrangements: left, wound bundle; right, leached bundle. 
It is crucial for OCT applications that light does not leak from any one fibre into neighbouring fibres. We have investigated this by coupling light into a single fibre and observing the output on a camera. At $800 \mathrm{~nm}$, leakage between neighbouring fibres was found to be negligible although, working with a wavelength of $1550 \mathrm{~nm}$, crosstalk was more significant.

The combination of coupling/transmission efficiency at $800 \mathrm{~nm}$ was measured to be $45 \%$. Coupling loss is low, due to the fact that the fibre bundles have high NA $(\mathrm{NA}=0.55)$ values and a mismatch is present with the external coupling optics. However, this leads to a significant optical power loss for returning beams. At $1550 \mathrm{~nm}$ the coupling/transmission efficiency was measured to be about $20 \%$. This is consistent with a higher mode field diameter and consequent leakage into neighboring fibres. Therefore a careful lens design is required at both input and output coupling system of lenses. The relatively large fibre diameter can lead to slight multi-moding of light in each fibre at $800 \mathrm{~nm}$ under certain launching conditions. The effect of multi-moding in the bundle would be that 'ghost' images would arise due to the different path length corresponding to each mode. This has not, however, been a problem in our experiments, and is even less probable at higher wavelengths having a larger mode field diameter. Pictures obtained by scanning the input beam across 3 individual fibres in succession are presented in figure 8a, together with a coherence envelope for one fibre in figure $8 \mathrm{~b}$.

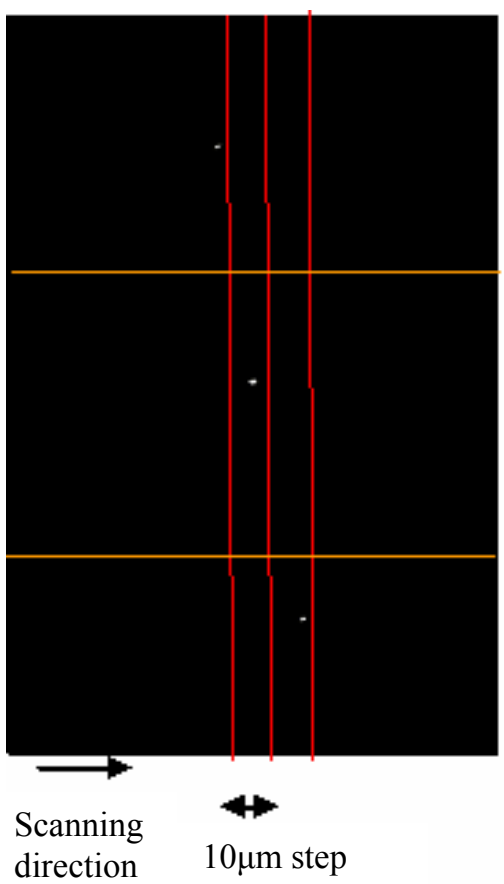

a)

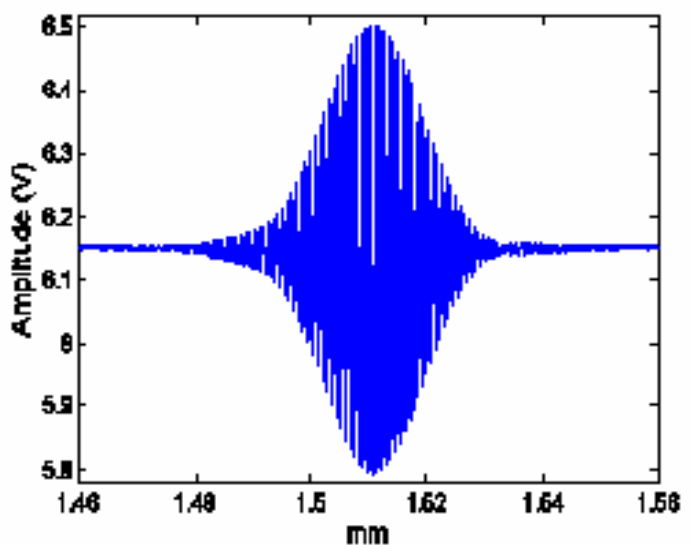

b)

Fig. 8. a) 3 fibres illuminated in sequence, by scanning of the input beam. b) Coherence envelope using mirror as sample. 


\subsection{Results and Discussion}

The bundle used was of the leached type as shown in figure 9. The bundle was scanned across the input beam in $10 \mu \mathrm{m}$ steps, and twenty fibres were addressed in succession.

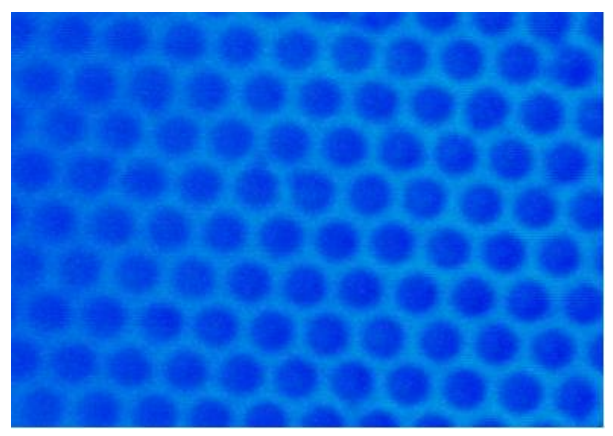

Fig. 9 Leached type bundle

A two-dimensional image was generated from two pieces of plastic projector slide stacked together as shown in figure 10. A-scan images were taken at each step position to construct an image of $0.2 \mathrm{~mm}$ (transverse) $\mathrm{x} 1 \mathrm{~mm}$ (depth).

The image produced is shown in figure 11. The image clearly depicts the four layers of the sample. The upper and lower surfaces of the plastic slides are clearly visible with an overall thickness of $0.157 \mathrm{~mm}$ for both slides. The air gap between them was found to be in the range of $0.471 \mathrm{~mm}$.

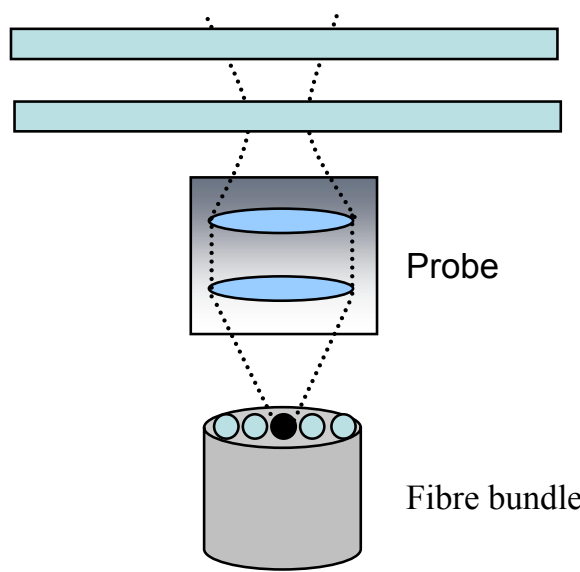

Plastic slides

Fig. 10. Experimental arrangement of the fibre bundle, probe and a sample of two plastic projector slides. 


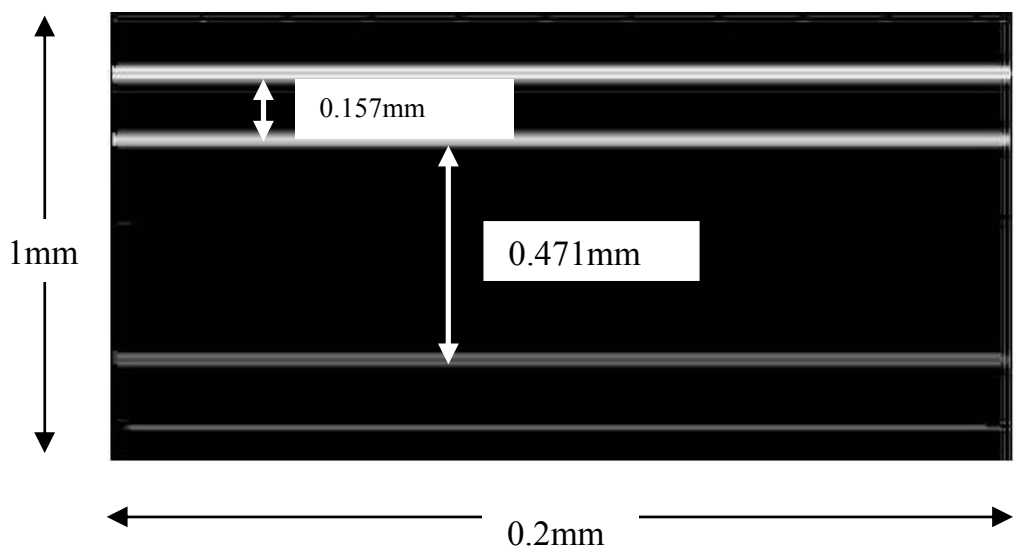

Fig. 11. Image of two layers of plastic projector slide.

\section{CONCLUSION}

A Fizeau configuration optical coherence tomography system has been developed, offering transverse and longitudinal spatial resolutions of around $20 \mu \mathrm{m}$. An SNR of $80 \mathrm{~dB}$ has been obtained from a mirror surface, and a series of oils of known index, deposited on glass, have been used to assess the SNR for interfaces of lower reflectivity. Implementation of a coherent fibre imaging bundle can enable 2D information to be obtained without the necessity for scanning components at the probe tip. The common path probe section of the instrument ensures 'downlead insensitivity' and, provided that the processing interferometer is constructed in bulk-optic form, the entire instrument is polarization insensitive. The use of an imaging bundle in a Fizeau interferometer based OCT would overall be advantageous as it will form an extremely compact, robust and downlead insensitive instrument for future endoscopic applications allowing 3D OCT image acquisition. Further development of the system will include testing on biological samples, miniaturisation of components, increased transverse scanning speed of the input beam across the bundle and its implementation in the Fourier domain rather than time domain.

\section{REFERENCES}

1. Izatt J A, Kulkarni M D, Wang H-W, Kobayashi $\mathrm{K}$ and Sivak M V Jr. "Optical coherence tomography and microscopy in gastrointestinal tissues" IEEE Journal of Selected topics in quantum electronics 2 1017-28 (1996)

2. Kersey A D, Marrone M J, Dandridge A and Tveten A B "Control scheme for polarization-induced signal fading in multiplexed optical fibre interferometric sensors" J. Lightwave Technol. 6 1599-1609 (1988)

3. Everett M J, Schoenerberger K, Colston B W and Da Silva L B "Birefringence characteristics of biological tissue by optical coherence tomography" Opt. Lett. 23 228-30 (1998)

4. Bamford K, James J, Barr H and Tatam R P "Radar detection of precancerous bronchial tissue "Lasers in Medical Science 15 188-94 (2000)

5. Beddows R, James S W and Tatam R P "Improved performance interferometer designs for optical coherence tomography" 15th International Conference on Optical Fibre Sensors (OFS-15) (Portland, Oregon, 6-10 May, 2002) IEEE Cat. No: 02EX533, 527-30 (2002)

6. Casaubieilh P, Ford H D and Tatam, R P "Optical coherence tomography with a Fizeau interferometer configuration" Proc. SPIE 5858-18 (2005) 
7. Sharma U, Kang J U and Fried N M 2005 "Fizeau optical coherence tomography: sensitivity optimization and system analysis" Conference on Lasers and Electro-Optics (CLEO) (San Jose, California, 22-27 May) Vol 3. 2061$2063(2005)$

8. Tumlinson A R, Barton J K, Povazay B, Sattman H, Unterhuber A, Leitgeb R A and Drexler W "Endoscope-tip interferometer for ultrahigh resolution frequency domain optical coherence tomography in mouse colon" Optics Express 14 1878-87 (2006)

9. Naci Inci M, Kidd S R, Barton J S and Jones J D C "Fabrication of single-mode fibre optic Fabry-Perot interferometers using fusion spliced titanium dioxide optical coatings" Meas. Sci Technol. 3 678-84 (1992)

10. Ford H D, Beddows R, Casaubieilh P and Tatam R P "Comparative signal-to-noise analysis of fibre-optic based OCT systems" Journal of Modern Optics 52 1965-1979 (2005)

11. Bouma, B.E., Tearney, G.J., Boppart, S.A., Hee, M.R., Brezinski, M.E.Fujimoto, J.G., Optics Letters, 20, 1486 (1995)

12. Colston, B.W., Everett, M.J., DaSilva, L.B., Otis, L.L., Stroeve, P. and Nathel, H., Applied Optics, 37, 3582 (1998)

13. T. Xie, H. Xie, G. K. Fedder, and Y. Pan, Electron. Letters. 39, 1535 (2003).

14. X. Li, C. Chudoba, T. Ko, C. Pitris, and J. G. Fujimoto,Optics. Letters, 25, 1520 (2000).

15. P. H. Tran, D. Mukai, M. Brenner, and Z. Chen, Optics.Letters. 29, 1236 (2004).

16. H. D. Ford, R. P. Tatam, Full-field optical coherence tomography using a fibre imaging bundle BiOS 2006, San Jose, USA, January (2006).

17. Leitgeb R, Hitzenberger C K and Fercher A " Performance of Fourier domain vs. time domain optical coherence tomography" Optics Express 11 889-94 (2003) 